OPEN ACCESS

Edited by:

Vito De Pinto,

University of Catania, Italy

Reviewed by:

Tuuli Käämbre,

National Institute of Chemical Physics

and Biophysics, Estonia

Catherine Brenner,

Délégation Paris-Villejuif-01, Center for the National Scientific Research

(CNRS), France

*Correspondence:

Eduardo N. Maldonado maldona@musc.edu

Specialty section:

This article was submitted to Mitochondrial Research,

a section of the journal

Frontiers in Physiology

Received: 16 July 2021

Accepted: 25 August 2021

Published: 29 September 2021

Citation:

Heslop KA, Milesi V and

Maldonado EN (2021) VDAC

Modulation of Cancer Metabolism:

Advances and Therapeutic

Challenges.

Front. Physiol. 12:742839.

doi: 10.3389/fphys.2021.742839

\section{VDAC Modulation of Cancer Metabolism: Advances and Therapeutic Challenges}

\author{
Kareem A. Heslop ${ }^{1}$, Veronica Milesi ${ }^{2}$ and Eduardo N. Maldonado ${ }^{1,3 *}$ \\ 'Department of Drug Discovery and Biomedical Sciences, Medical University of South Carolina, Charleston, SC, United States, \\ ${ }^{2}$ Facultad de Ciencias Exactas, Instituto de Estudios Inmunológicos y Fisiopatológicos (IIFP), UNLP, CONICET, \\ CIC PBA, La Plata, Argentina, ${ }^{3}$ Hollings Cancer Center, Medical University of South Carolina, Charleston, SC, United States
}

Most anionic metabolites including respiratory substrates, glycolytic adenosine triphosphate (ATP), and small cations that enter mitochondria, and mitochondrial ATP moving to the cytosol, cross the outer mitochondrial membrane (OMM) through voltage dependent anion channels (VDAC). The closed states of VDAC block the passage of anionic metabolites, and increase the flux of small cations, including calcium. Consequently, physiological or pharmacological regulation of VDAC opening, by conditioning the magnitude of both anion and cation fluxes, is a major contributor to mitochondrial metabolism. Tumor cells display a pro-proliferative Warburg phenotype characterized by enhanced aerobic glycolysis in the presence of partial suppression of mitochondrial metabolism. The heterogeneous and flexible metabolic traits of most human tumors render cells able to adapt to the constantly changing energetic and biosynthetic demands by switching between predominantly glycolytic or oxidative phenotypes. Here, we describe the biological consequences of changes in the conformational state of VDAC for cancer metabolism, the mechanisms by which VDAC-openers promote cancer cell death, and the advantages of VDAC opening as a valuable pharmacological target. Particular emphasis is given to the endogenous regulation of VDAC by free tubulin and the effects of VDAC-tubulin antagonists in cancer cells. Because of its function and location, VDAC operates as a switch to turn-off mitochondrial metabolism (closed state) and increase aerobic glycolysis (pro-Warburg), or to turn-on mitochondrial metabolism (open state) and decrease glycolysis (anti-Warburg). A better understanding of the role of VDAC regulation in tumor progression is relevant both for cancer biology and for developing novel cancer chemotherapies.

Keywords: cancer, glycolysis, metabolic flexibility, metabolic reprogramming, metabolism, mitochondria, voltage dependent anion channels, Warburg

\section{INTRODUCTION}

The relative contribution of aerobic glycolysis and oxidative phosphorylation (Oxphos) to overall ATP generation, determine cancer bioenergetics. Cancer metabolism, however, involves not only chemical reactions to cope with energy demands, but also those necessary to maintain anabolism and catabolism. The study of cancer metabolism preceded the discovery of oncogenes 
and tumor suppressors by approximately 50 years, becoming one of the oldest areas of research in cancer biology. A different metabolism in tumor cells, compared to non-proliferating cells, is regarded essential to develop and maintain malignant characteristics. A "reprogrammed metabolism," considered by many a hallmark of cancer and observed quite generally across many types of tumor cells, is a major driver of tumor metabolism (Cerezo and Rocchi, 2020; Faubert et al., 2020). Metabolic reprogramming, induced by oncogenic mutations among other factors, refers to the enhancement or suppression of specific metabolic pathways in tumor cells that improve the cellular fitness required for rapid cell division. Despite its relevance for long-term cell survival, reprogramming is not sufficient to explain metabolic adaptations to fast changing demands. It is very likely that rapid metabolic changes be driven by fast acting mechanisms that modulate specific pathways or proteins not depending on reprogramming. In particular, regulation of the conductance of voltage dependent anion channels (VDAC), located in the outer mitochondrial membrane (OMM) will be described here as a mechanism potentially involved in fast metabolic responses.

Regardless of the relatively long history of research on cancer metabolism, the interest on the role of mitochondrial metabolism in tumors was limited until the end of the 20th century. A 2021 updated PubMed search using the words mitochondrial metabolism, or mitochondrial metabolism and cancer, showed only $\sim 33$ and $12 \%$ of the total publications during the period 1921-2000. The number of indexed publications for both topics exponentially increased in the last 2 decades. Even more strikingly, another search using the words VDAC and cancer, showed only $\sim 3 \%$ of the total publications, reported from 1986 to 2000 .

The relevance of mitochondria for cellular metabolism started to be unveiled in the early 20th century, when oxidations in the mitochondrial matrix and ATP synthesis, were identified as main functions of the organelle. However, a mechanistic explanation reconciling observation with a theory to explain the link between oxidation and ATP synthesis was lacking. The chemiosmotic hypothesis of Mitchell (1966) solved the problem by postulating a proton electrochemical gradient across the inner mitochondrial membrane (IMM) as the energy-rich intermediate of Oxphos (Mitchell and Moyle, 1967). Mitchell (1966) proposed that the flow of electrons through complexes of the electron transport chain (ETC) was coupled to the outward translocation of $\mathrm{H}^{+}$across the IMM creating a proton motive force $(\Delta \mathrm{p})$ used by the ATP synthase to synthesize ATP from adenosine diphosphate (ADP) and inorganic phosphate $(\mathrm{Pi})$. The hypothesis of Mitchell (1966) was later confirmed by experimentation in mitochondria, chloroplasts, and bacteria.

A major trigger to research in cancer metabolism were the seminal findings made in the early 20th century by the German biochemist Otto Warburg, who showed in tumor slices and ascites cancer cells, that tumors produce more lactic acid than non-tumor cells even at physiological partial pressures of oxygen (Warburg et al., 1927; Warburg, 1930). This particular phenotype, called Warburg effect, is characterized by enhanced aerobic glycolysis. In tumor cells, relatively low cytosolic ATP/ADP ratios caused by a partial suppression of mitochondrial metabolism prevents glycolysis inhibition mediated by high ATP. Warburg (1956) even proposed that damaged mitochondria were the origin of cancer. According to his hypothesis, only those cells with irreversible but incomplete damage to respiration capable of increasing the conversion of glucose to lactic acid (fermentation) become cancerous. The provocative hypothesis of failing mitochondria as the origin of cancer was quickly challenged by Weinhouse (1956) and others, who demonstrated both high glycolysis and oxidative metabolism in cancer tissues. In fact, all tumors display a certain level of enhanced glycolysis that always coexists with functional mitochondria. Measurements of oxygen consumption, mitochondrial membrane potential $(\Delta \Psi)$, 1,4-dihydronicotinamide adenine dinucleotide (NADH) production, and ATP generation, among other parameters, have confirmed that tumor mitochondria are metabolically active (Nakashima et al., 1984; Maldonado et al., 2010; Mathupala et al., 2010; Lim et al., 2011; Moreno-Sanchez et al., 2014; Singleterry et al., 2014). Variations in the relative contribution of mitochondrial ATP to cellular bioenergetics among different tumor types and even within the same tumor, suggest a dynamic regulation of oxidative metabolism.

In this review, we will describe the biological effects induced by changes in the conformational states of VDAC; how an increase of VDAC conductance reverses the Warburg phenotype and promotes cell death; and finally, the relevance of VDAC as a pharmacological target to develop novel cancer chemotherapies.

\section{VDAC STRUCTURE AND REGULATION OF CONDUCTANCE}

The discovery of VDAC in mitochondrial extracts from Paramecium tetraurelia, followed by the identification in mammalian cells, opened a new avenue in the research and understanding of mitochondrial metabolism (Schein et al., 1976; Colombini, 1979). VDAC, comprising three isoforms, is a polypeptide of $\sim 30 \mathrm{kDa}$ (VDAC 1 and 3: 280 amino acids; VDAC 2: 291 amino acids). In most mammalian cells, including cancer cells, VDAC1 and 2 are the most abundant isoforms, whereas VDAC3 is the least expressed, except for testis and spermatozoa (Sampson et al., 1997; De Pinto et al., 2010). VDAC $\beta$-barrels enclose an aqueous channel of $\sim 3 \mathrm{~nm}$ internal diameter in the open state, that allows the passage of molecules up to $\sim 5 \mathrm{kDa}$ (Colombini, 1980, 2012; Mannella, 2021). The current consensus about VDAC structure shows differences with the originally proposed model by Colombini that was based on biochemical and functional data (Colombini, 2009, 2012). Structural studies using NMR and X-ray crystallography have shown VDAC1 and VDAC2 as a transmembrane $\beta$-barrel protein with $19 \beta$-strands, mostly anti-parallel, except for strands 1 and 19. Both isoforms also have an $\mathrm{N}$-terminal, $\alpha$-helical region located within the pore (Ujwal et al., 2008; Hiller et al., 2010). 
Voltage dependent anion channels inserted in non-polarized or weakly polarized membranes (close to $0 \mathrm{mV}$ ), stays mostly in the high conductance open state. By contrast, positive or negative membrane potentials induce conformational changes to several lower conductance closed states (maximal at -45 or $+45 \mathrm{mV}$; Bowen et al., 1985; Colombini, 1989). Although, it is currently not possible to determine membrane potentials across the OMM in live cells, plausible theoretical approaches suggest the existence of polarization of the OMM in intact cells (Lemeshko, 2021). Moreover, the $\sim 0.6 \mathrm{pH}$ difference reported between the cytosol and the mitochondrial intermembrane space (IMS) (Porcelli et al., 2005), corresponds to a $-15-20 \mathrm{mV}$ potential. Since this value of membrane potential falls in the range in which reconstituted VDAC display a mild decrease in conductance (Zizi et al., 1998), it is theoretically possible that voltage actually contributes to the regulation of VDAC opening.

Voltage dependent anion channels are selective for anionic metabolites and small cations, as showed initially by Colombini, and later confirmed by other groups (Choudhary et al., 2010; Villinger et al., 2014; Colombini, 2016). The open state of VDAC allows the flux of anions, including most respiratory substrates, $\mathrm{ATP}^{4-}, \mathrm{ADP}^{3-}, \mathrm{HPO}^{2-}$, phosphocreatine ${ }^{2-}$, and AMP, among others. In the closed state, VDAC favors a non-selective flux of cations including $\mathrm{Na}^{+}, \mathrm{K}^{+}$, and $\mathrm{Ca}^{2+}$ (Tan and Colombini, 2007; Colombini, 2012; Sander et al., 2021). VDAC closure, induced by voltage, increases the flux of $\mathrm{Ca}^{2+}$ up to 10 -fold (Tan and Colombini, 2007). Moreover, the magnitude of $\mathrm{Ca}^{2+}$ flux through VDAC is influenced by the type and amount of each isoform present (only VDAC1 and 2 seem involved in $\mathrm{Ca}^{2+}$ signaling); post-translational modifications (phosphorylation and monoubiquitinylation); and possibly interactions with partner proteins including $\mathrm{Bcl}-\mathrm{xL}$ and translocator protein (TSPO), among others (Sander et al., 2021). However, a higher flux of calcium through VDAC in mitochondria of intact cells does not necessarily correlates with an increase in the $\mathrm{Ca}^{2+}$ content in the matrix. After entering the IMS, $\mathrm{Ca}^{2+}$ still needs to be transported through the IMM by the mitochondrial calcium uniporter holocomplex (Fan et al., 2020). Thus, mitochondrial uptake of $\mathrm{Ca}^{2+}$ is subjected to multiple levels of regulation both at the OMM and the IMM. It remains to be determined experimentally if VDAC-mediated increase in the flux of $\mathrm{Ca}^{2+}$, actually influences $\mathrm{Ca}^{2+}$ content in the mitochondrial matrix modifying mitochondrial metabolism.

Overall, every physiological or pharmacological regulation of VDAC to induce a change from the open state to the closed states, reduces or increases the flux of negatively charged metabolic substrates and cations, respectively. Although gating and selectivity for VDAC1 and VDAC2 are very similar in different cell types, the detailed molecular determinants of voltage gating are still incompletely understood. A structural model proposes that the N-terminus of VDAC1 lying inside the pore, parallel to the wall, moves to the lumen blocking the passage of metabolites (Shuvo et al., 2016). Regardless of the mechanism controlling gating, the flux of polar metabolites through VDAC is determined mostly by their charge and size (Colombini, 1980, 2004).
Even though VDAC was initially considered constitutively open, like an "all-time open gateway," subsequent research both in vitro and in intact cells, showed regulation of VDAC conductance by several endogenous molecules. VDAC conductance has been shown to be modulated by $\alpha / \beta$ tubulin heterodimers (Rostovtseva et al., 2008; Timohhina et al., 2009; Maldonado et al., 2013); hexokinase (Pastorino et al., 2002; Al Jamal, 2005); bcl2 family members (Tsujimoto and Shimizu, 2000); glutamate (Gincel et al., 2001); and NADH (Zizi et al., 1994). It has also been demonstrated that post-translational modifications, mainly phosphorylation by protein kinases, GSK3 $\beta$, PKA, and protein kinase C epsilon (PKCe), blocks or inhibits association of VDAC with other proteins, such as Bax and tBid, and regulates VDAC opening (Heiden et al., 2001; Baines et al., 2007; Das et al., 2008). Moreover, PKA-dependent VDAC phosphorylation and GSK3 $\beta$-mediated VDAC2 phosphorylation increase VDAC conductance and also the sensitivity to tubulin inhibition (Bera et al., 1995; Das et al., 2008; Sheldon et al., 2011). VDAC opening is also modulated by protein-protein interactions with actin, p53, mitochondrial creatine kinase, and alpha-synuclein, among others (Rovini, 2019; Kanwar et al., 2020).

Overall, the movement of metabolites through VDAC dynamically depends on the concentration gradient of each permeant molecule reaching the OMM, the electric field, the number of functional VDAC channels, the selectivitypermeability to a particular metabolite, and the open probability of the channel.

\section{VDAC OPENING, MITOCHONDRIAL METABOLISM, AND WARBURG EFFECT}

VDAC1, VDAC2, and VDAC3, at the interphase between mitochondria and cytosol, are strategically located to control the flux of metabolites and ATP entering or leaving mitochondria. At present, it is unclear if the flux of metabolites and nucleotides through VDAC is different among tumor cell types, and if it is isoform specific. To access the mitochondrial matrix, most anionic substrates that cross the OMM only through VDAC, are further transported through the IMM by several finely tuned specific carriers (Palmieri and Pierri, 2010). Pyruvate, fatty acids, and the amino acids glutamine (quantitatively the most important amino acid utilized by several tumors), glycine, serine, leucine, isoleucine, valine, and tryptophan, generate acetyl-coenzyme A ( $\mathrm{AcCoA})$ that fuels the Krebs (tricarboxylic acid) cycle. A cycle of oxidation generates $\mathrm{NADH}$, and dihydroflavine-adenine nucleotide $\left(\mathrm{FADH}_{2}\right)$, electron donors to the ETC. Electrons flowing through the ETC or respiratory chain, formed by complexes I-IV, reduce the final acceptor molecular $\mathrm{O}_{2}$ to $\mathrm{H}_{2} \mathrm{O}$, while simultaneously generating single electron leaks from complexes I, II, and III to form the superoxide anion $\left(\mathrm{O} 2^{*}\right)$, that is further converted into other reactive oxygen species (ROS) (Chance et al., 1979; Mailloux, 2020). The metabolic fitness of mitochondria in any cell type, also depends on the generation of $\Delta \Psi$ formed when complexes I, III, and IV drive $\mathrm{H}^{+}$translocation from the matrix to the 
IMS, where it generates a negative transmembrane $\Delta \Psi$ and a $\Delta \mathrm{pH}$, both components of the proton motive force $(\Delta \mathrm{p})$. Ultimately, $\Delta \mathrm{p}$ drives ATP synthesis from $\mathrm{ADP}$ and $\mathrm{Pi}$ by complex V ( $\mathrm{F}_{1} \mathrm{~F}_{\mathrm{O}}$-ATP synthase): (Hoek et al., 1980; Nicholls and Ferguson, 2013). Newly synthesized ATP is transported to the cytosol through the adenine nucleotide translocator, located in the IMM, and exchanged for ADP in a 1:1 molar ratio to be finally released to the cytosol through VDAC (Klingenberg, 2008; Allouche et al., 2012). AMP, another adenine nucleotide, also cross the OMM through VDAC. Therefore, regulation of VDAC opening influences the ATP/AMP ratio, which in turn, modulates the activation of AMP-activated protein kinase (AMPK); (Colombini, 2016; Shevade et al., 2018). Thus, the magnitude of metabolite fluxes to support Oxphos and ATP synthesis, ultimately depends on VDAC conductance (Figure 1).

In general, approximately $95 \%$ of ATP in quiescent cells is produced by Oxphos, with the remaining 5\% generated through the pay-off phase of glycolysis in the cytosol, and the succinyl-CoA ligase reaction of the Krebs cycle. Full mitochondrial oxidation of glucose generates $\sim 32$ moles of ATP, as estimated by different methods, compared to the 2 moles of ATP/mole of glucose during glycolysis (Brand, 2005). Although the total amount of mitochondrial ATP calculated considers the currently accepted proton stoichiometry for ATP synthesis, ATP/ADP-Pi exchange, respiration, and the malate/aspartate shuttle, the actual ATP yield could be less due to proton leak into the mitochondrial matrix (Brand, 2005; Rich and Marechal, 2010; Walker, 2013; Wikstrom et al., 2015). The relatively low ATP demand for cell division compared to the energy requirements for maintenance of cellular functions, mainly the activity of the $\mathrm{Na}^{+}-\mathrm{K}^{+}$ATPase, suggests that ATP generation is not a limiting factor to sustain rapid cell proliferation (Kilburn et al., 1969; Schwenke et al., 1981; Veech et al., 2019; Seyfried et al., 2020). In non-proliferating cells, high VDAC conductance promotes an oxidative metabolism, generating cytosolic ATP/ADP ratios 50-100 times higher compared to mitochondria. High ATP-ADP in the cytosol inhibits phosphofructokinase-1 (PFK-1), a rate limiting step in the glycolytic pathway, among other possible mechanisms blocking glycolysis (Schwenke et al., 1981; Jenkins et al., 2011). The ATP-dependent inhibitory mechanisms, together with regulation of other pathways, may be key to explain the reciprocal dependence between mitochondrial metabolism and glycolysis in several tumor types. By contrast, a partial suppression of mitochondrial metabolism in cancer cells contributes to a low cytosolic ATP/ ADP ratio; releasing the brake on glycolysis and favoring the Warburg phenotype. Compared to non-proliferating cells, cancer cells generate $\sim 10-90 \%$ of total ATP by glycolysis (Nakashima et al., 1984; Griguer et al., 2005). Tumor cells also display "glucose avidity," an increased uptake of glucose compared to non-proliferating cells. In clinical settings, the preferential incorporation of the radioactive glucose analog fluorodeoxyglucose by tumor cells is used in positron emission tomography (PET) to diagnose cancer (Zhu et al., 2011). A downside of PET scan that leads to false positives is the inability to distinguish between tumor cells and non-tumor cells with a high rate of glucose uptake, including tumor infiltrating lymphocytes.

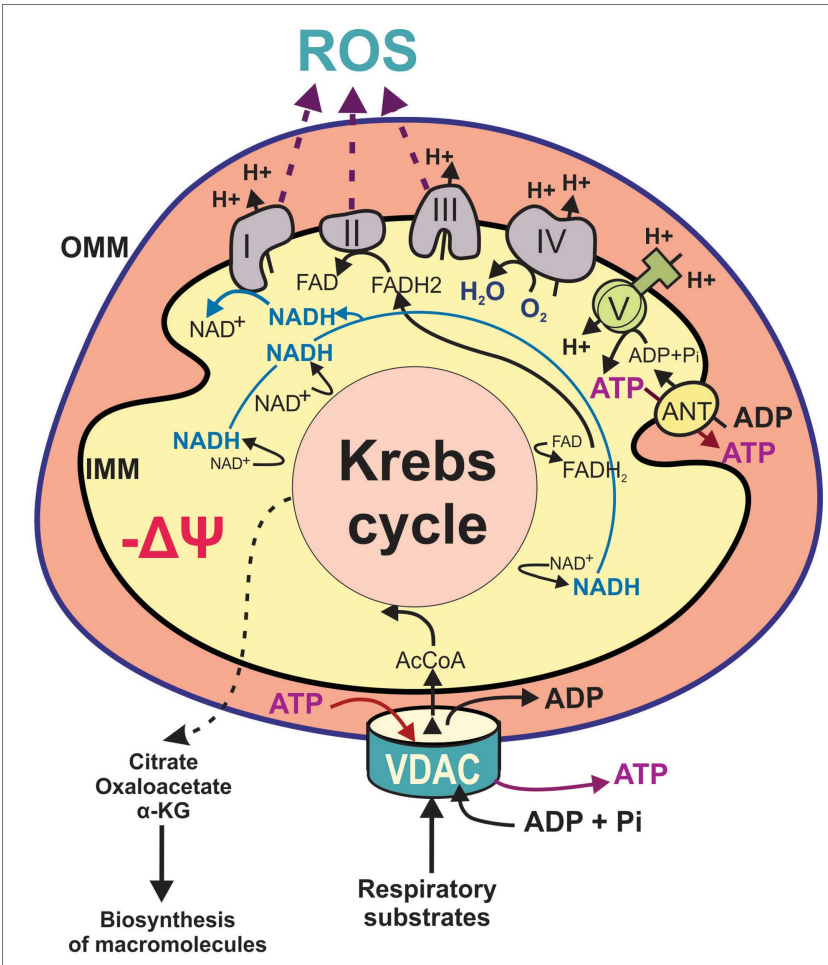

FIGURE 1 | Main features of mitochondrial metabolism. Flux of metabolites including the respiratory substrates pyruvate, adenosine diphosphate (ADP), and inorganic phosphate $(\mathrm{Pi})$ and certain amino acids, cross the outer mitochondrial membrane occurs through voltage dependent anion channels (VDAC). Catabolism of respiratory substrates in the Krebs cycle generates 1,4-dihydronicotinamide adenine dinucleotide (NADH) and dihydroflavineadenine nucleotide $\left(\mathrm{FADH}_{2}\right)$, which fuel the electron transport chain (ETC; complexes I-IV) to support oxidative phosphorylation. The Krebs cycle also produces metabolic intermediaries released to the cytosol for the biosynthesis of proteins and lipids. Proton pumping by the respiratory chain across inner mitochondrial membrane (IMM) generates mitochondrial $\Delta \Psi$. Protons moving back across MIM into the matrix drive ATP synthesis from ADP and Pi by the $F_{1} F_{0}$-ATP synthase (complex V). Mitochondrial ATP is exported from the matrix by the adenine nucleotide transporter (ANT) and released to the cytosol through VDAC. Flow of electrons through complexes I, II, and III also generates reactive oxygen species. AcCoA, acetyl CoA; $\alpha-\mathrm{KG}$, alpha ketoglutarate; IMM, inner mitochondrial membrane; OMM, outer mitochondrial membrane; and ROS, reactive oxygen species.

The conformational open or closed state of VDAC, by regulating the flux of respiratory substrates, is a major determinant of cytosolic ATP/ADP ratios to favor or oppose the pro-proliferative Warburg phenotype. The predominance of open or closed conformational states of VDAC, not only determines the bioenergetics efficiency of mitochondria, but also the ability to contribute metabolic intermediaries to the synthesis of amino acids, fatty acids, nucleotides, cholesterol, glucose, and heme (Spinelli and Haigis, 2018). Rapidly dividing cells face a constant challenge to produce new macromolecules to approximately double the biomass before mitosis. In the Warburg metabolism, the major sources of carbon backbones are glucose, glutamine, and fatty acids. Similar to glycolytic intermediates, Krebs cycle intermediates are also used as precursors for biosynthesis of macromolecules. A continuous 
supply of precursors, sustained by anaplerotic pathways, "refills" the cycle with intermediates to replace those that have been already used for biosynthesis. Glutaminolysis, which produces $\alpha$-ketoglutarate from glutamine, and pyruvate carboxylation, which produces oxaloacetate from glucose/pyruvate are major contributors to anaplerotic fluxes in cancer cells (Bott et al., 2019; Martins et al., 2020; Kiesel et al., 2021). Oxidation of the branched-chain amino acids (BCAAs) isoleucine and valine also provides an anaplerotic flux in some tumors. If glucose supply is sufficient for energy generation, glutamine-derived $\alpha$-ketoglutarate and oxaloacetate are utilized for the synthesis of nonessential amino acids, whereas citrate exported to the cytosol, is converted into AcCoA and utilized for the synthesis of fatty acids, cholesterol, and amino acids. Glutamine is also a nitrogen donor in purine and pyrimidine synthesis, and a precursor for the synthesis of the antioxidant glutathione (Owen et al., 2002; Still and Yuneva, 2017; Li and Le, 2018; DeBerardinis and Chandel, 2020). Recently, one-carbon metabolism, a set of reactions that transfer one-carbon units (methyl groups) from serine and glycine, has been shown to be important for de novo synthesis of purines and thymidylate synthase in highly proliferative tumors (Meiser and Vazquez, 2016).

Overall, VDAC operates as a biological switch that, in the on-phase (open state), maximizes the flux of metabolites for optimal mitochondrial function, whereas during the off-phase (closed state), minimizes mitochondrial metabolism (Figure 2). Thus, regulation of only this channel has an amplifying effect on several intra and extra-mitochondrial pathways modulating cancer metabolism and bioenergetics. The dynamic changes in ATP and biosynthesis demands, ranging from seconds to minutes or even hours, imply the coexistence of both metabolic reprogramming and fast acting regulatory mechanisms. VDAC is, very likely, one of the rapidly adapting mechanisms that are responsive to interactions with other OMM and cytosolic proteins and soluble factors, along with transient or permanent posttranslational modifications. At present, it is unknown if metabolic reprogramming, that affects several mitochondriarelated pathways, modulates VDAC conductance.

Beyond the role in mitochondrial metabolism, VDAC is also a prognostic biomarker for certain types of human cancer (Jozwiak et al., 2020; Wersall et al., 2021). High expression of VDAC1 has been associated with unfavorable outcomes in cancers from lung, head and neck, breast, and liver (Grills et al., 2011; Yang et al., 2019; Jozwiak et al., 2020). High transcript levels of VDAC2 in multiple tumors, including melanoma, epithelial thyroid tumors, and breast cancer have been reported in the cancer databases cBioportal, ${ }^{1}$ and the Human Protein Atlas. ${ }^{2}$ In head and neck cancer and in liver cancer, high expression of VDAC2 is also associated with a poor outcome. Interestingly, VDAC3 expression seems not to be of prognostic value for human cancer suggesting isoform specific effects on cell proliferation.

${ }^{1}$ http://www.cbioportal.org

${ }^{2} \mathrm{http}: / /$ www.proteinatlas.org

\section{MITOCHONDRIA, TUMOR METABOLIC FLEXIBILITY, AND TUMOR HETEROGENEITY}

The term metabolic inflexibility, coined in the late 90's, refers to inadequate responses of skeletal muscle to fuel changes in insulin-resistant obese patients (Kelley and Simoneau, 1994; Kelley et al., 1999). By contrast, metabolic flexibility alludes to the ability of muscle cells to switch from fatty acid to glucose oxidation. A "mitocentric" concept of nutrient metabolism describes the storage, utilization, and conversion of nutrients into other metabolites, as a critical process to monitor energy homeostasis (Trepanowski et al., 2011; Gambardella et al., 2020; Motori et al., 2020). Research to identify the molecular origins of metabolic flexibility has focused mostly on the interplay between glucose and fatty acids, and/or the aberrant production of the fatty acid precursor, malonyl-CoA. Mitochondria, as an integral metabolic hub, are major contributors to metabolic flexibility. Similar to muscle cells, most cancer cells are metabolically flexible. The different quantitative contributions of mitochondria to cellular bioenergetics, together with genomic instability and differences in the microenvironment, are important determinants of tumor heterogeneity (Kuipers et al., 2017; Barcena-Varela and Lujambio, 2021; Wei et al., 2021). Tumor metabolic heterogeneity is increasingly recognized as a factor causing failures in cancer treatment (Gentric et al., 2017; Kim and DeBerardinis, 2019). Whether a tumor displays a predominantly glycolytic or oxidative metabolism depends on gene expression as well as temporary and long-term epigenetic stimuli. In addition, the dynamic relative contribution of glycolysis and Oxphos is influenced by the type of primary or metastatic tumor, intra-tumor regional differences, temporal variations in the energetic demands and availability of glucose, fatty acids, ketone bodies, and certain amino acids (Jose et al., 2011; Alam et al., 2016; Duraj et al., 2021; Figure 2).

A reciprocal dependence of mitochondrial metabolism and enhanced glycolysis has been shown in several cancer cell types under different experimental conditions, including hypoxia and limitations in the availability of nutrients. The magnitude of Oxphos inhibition during hypoxia is influenced by the cell type and duration of the hypoxic exposure. Prolonged hypoxia increases glycolysis in MCF-7 cells but not in HeLa cells, although, Oxphos is the predominant source of ATP in both cell types (Rodriguez-Enriquez et al., 2010). Interestingly, in solid tumors, the respiratory chain is still fully functional at oxygen levels as low as $0.5 \%$, indicating that cancer cells exposed to $<2 \%$ oxygen in rapidly growing and heterogeneously perfused tumors still produce ATP by Oxphos (Vaupel et al., 2001; McKeown, 2014). In some human cell models, hypoxia induced the synthesis of a C-terminal truncated form of VDAC1, with similar channel activity and voltage dependency as the fulllength channel. Truncated VDAC1 was linked to an upregulation of both Oxphos and glycolysis, as well as to resistance to apoptosis (Brahimi-Horn et al., 2012; Brahimi-Horn and Mazure, 2014; Mazure, 2016; Cunha-de Padua et al., 2020). By contrast, knockout of VDAC1 in mouse embryonic fibroblasts (MEF) 


\section{A}

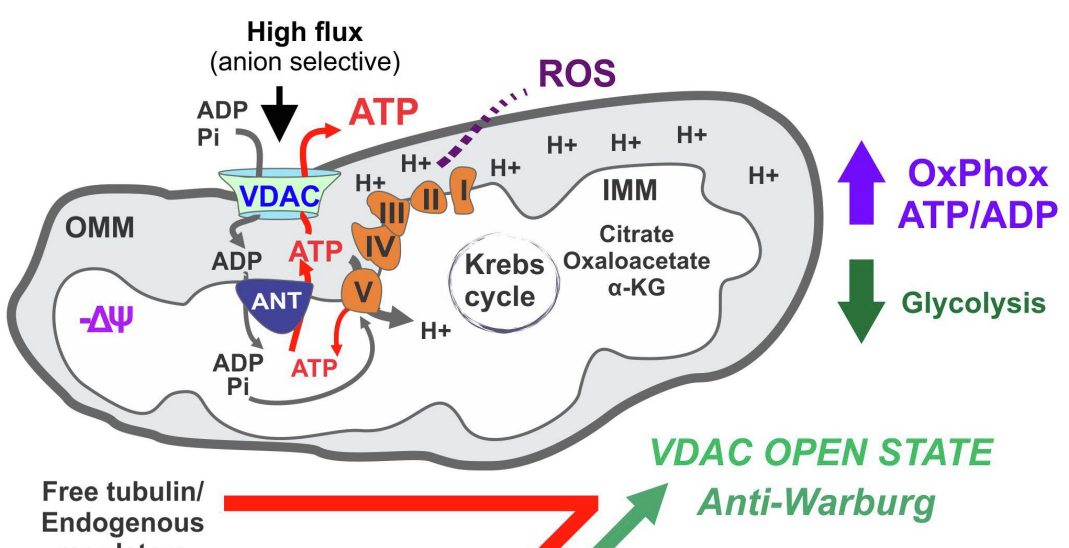
regulators

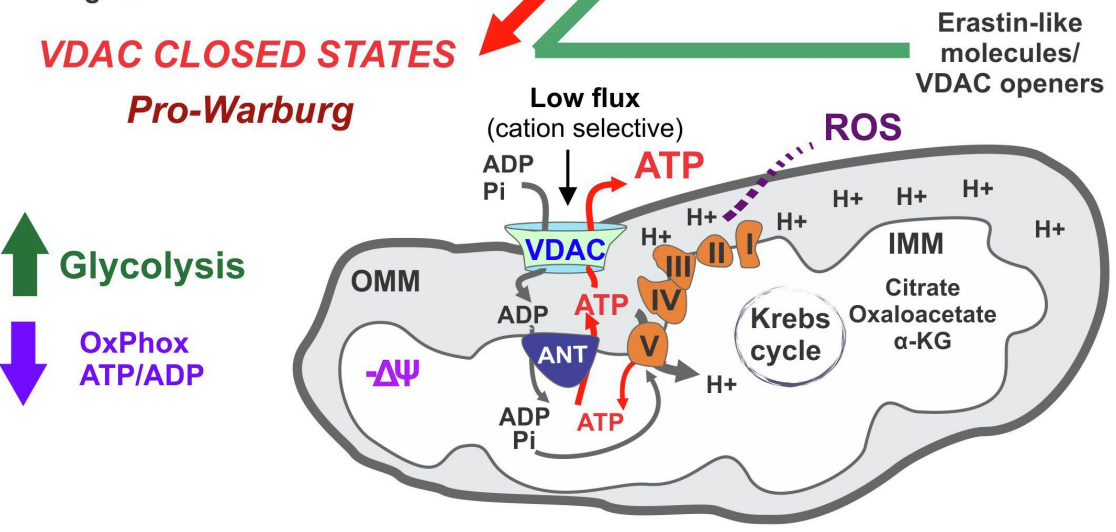

B
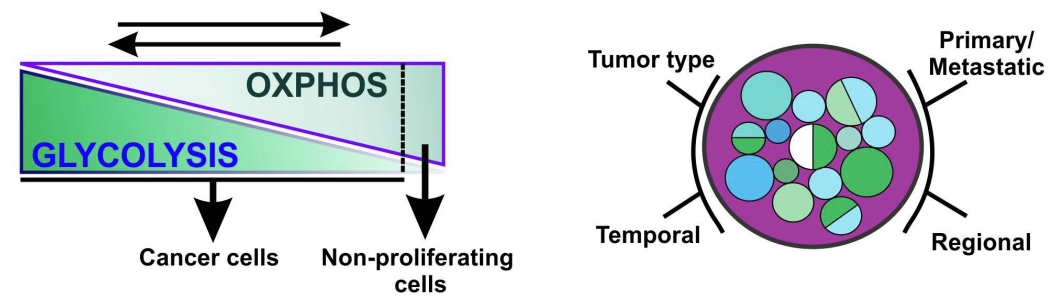

FIGURE 2 | Voltage dependent anion channels regulation of cancer bioenergetics in metabolically flexible tumors. VDAC opening in cancer cells promotes oxidative metabolism and reverses the Warburg phenotype (A). The contribution of glycolysis to cancer cell bioenergetics is influenced by the type of tumor, and regional differences, among other variables. A dynamic reversal of the Warburg phenotype influences cell proliferation (B).

expressing oncogenic RAS, favors tumor development in mice by promoting metabolic reprogramming (Brahimi-Horn et al., 2015). Beyond hypoxia, under conditions that decrease pyruvate oxidation in the Krebs cycle, mitochondria from tumor cells adapt to oxidize more glutamine as an energy source sustaining tumor growth both through aerobic glycolysis and Oxphos (Mullen et al., 2012). Nutrient availability not only influences tumor growth but also induces a switch from aerobic glycolysis to Oxphos in lymphoma and breast cancer cell lines cultured in glucose-free media (Smolkova et al., 2010; Robinson et al., 2012). If access to glucose and glutamine is limited, tumor cells adapt to utilize instead, lactate, methionine, arginine, cysteine, asparagine, leucine, acetate, and even lipids and proteins from the microenvironment to cope with the energy demands (Kreis et al., 1980; Clavell et al., 1986; Scott et al., 2000;
Commisso et al., 2013; Kennedy et al., 2013; Comerford et al., 2014; Keenan and Chi, 2015). Overall, tumors display several metabolic alternatives to support continuous cell division regardless of unfavorable environmental conditions.

\section{VDAC AS A PHARMACOLOGICAL TARGET}

Conventional chemotherapeutic agents promote cell death or arrest cell proliferation by blocking DNA synthesis and replication, inhibiting specific enzymes or receptors, or by destabilizing or stabilizing microtubules. By contrast, "metabolic" approaches for cancer treatment have mostly focused on the inhibition of glycolysis. Only more recently, mitochondria and mitochondrial 
metabolism emerged as a source of targets to prevent or slow tumor progression (Adachi et al., 2004; Doherty and Cleveland, 2013; Bhat et al., 2015; Weinberg and Chandel, 2015; Fang and Maldonado, 2018; Curcio et al., 2021). In general, a restriction of mitochondrial oxidation of substrates decreases the amount of each intermediary released to the cytosol for the biosynthesis of macromolecules. By contrast, enhancement of mitochondrial metabolism leads to increased oxidation of substrates and ATP generation, increased ROS formation with subsequent oxidative stress, and reversal of the Warburg phenotype. These two ways of modulating mitochondrial metabolism decrease cell proliferation and promote cell death (Figure 3).

A remarkable and unique feature of VDAC is the ability to globally control mitochondrial metabolism by simply increasing or decreasing the conductance. In a broad sense, VDAC is a "first-step" in mitochondrial metabolism. Although, mitochondrial bioenergetics is regulated at multiple levels, VDAC opening in cancer cells leads to three major biological effects: maximization of full oxidation because of augmented entry of substrates into mitochondria; subsequent decrease in glycolysis due to a high cytosolic ATP/ADP ratio promoted by maximum generation of mitochondrial ATP; and increased formation of ROS following the enhanced activity of the ETC. Particularly relevant for cancer metabolism, VDAC has been shown to also serve as a docking site for a group of cytosolic proteins including hexokinase-II and the Bcl-2 family of proteins. While VDAC1 is considered a proapoptotic protein and VDAC2 seems to be anti-apoptotic, some controversy still remains about the role of VDAC for apoptotic cell death (Camara et al., 2017; Shoshan-Barmatz et al., 2017; Chin et al., 2018). Regardless, because of the obvious relevance of apoptosis for tumor growth, VDAC1 has emerged as a candidate to develop VDAC1-targeting molecules. Several peptides (mastoparan, mitoparan, and TEAMVP); an oligonucleotide (G3139); molecules of unrelated structures (dicyclohexylcarbodiimide, estradiol, among several others); and more recently a miRNA (miR-7), have been reported to target VDAC and be pro or anti-apoptotic. These different molecules either regulate VDAC1 activity, expression, oligomerization, and interaction with HK-II or posttranslational modifications. An excellent review providing a brief description of compounds interacting mostly with VDACl, have been published by De Pinto group (Magri et al., 2018).

Regardless of older and recent developments including a resolved NMR structure of VDAC1 with NADH bound (Bohm et al., 2020), and the identification of a cholesterol binding site (Budelier et al., 2017), drug discovery of VDAC modulators still faces major challenges. Despite the growing number of compounds that have an effect on VDAC, potential isoform specificities and identification of ligand binding sites for each of the reported molecules, are still unknown. An analysis of the structures of anti-cancer drugs acting on VDAC shows a lack of common structural motifs. In addition, a well-defined druggable binding site has not been yet identified. To add more complexity, and even when electrophysiology of VDAC inserted into lipid membranes is likely the best method currently available to study the effect of a molecule on VDAC conductance, the results may not be definitive. A different lipid composition of the artificial membranes compared to the OMM, lack of cytosolic soluble factors and protein interactions, limited knowledge about the effect of posttranslational modifications, potential artifacts introduced during the isolation and insertion of VDAC, as well as isoform specific responses, may lead to inconclusive results. Moreover, a compound of interest could eventually affect VDAC gating inserted into bilayers not only by interacting directly or indirectly with the channel, but also by altering the lipid bilayer surrounding the channel (Rostovtseva et al., 2020).

In the last 10 years, we showed that VDAC regulates mitochondrial metabolism in live cancer cells using a combination of knockdown strategies and confocal microscopy of $\Delta \Psi$ and $\mathrm{NADH}$, among other techniques. We also provided evidence that increased cytosolic free tubulin dynamically correlates with changes in $\Delta \Psi$, suggesting a direct effect of free tubulin on VDAC opening (Maldonado et al., 2013). Our initial findings about the effect of VDAC regulation by free tubulin on mitochondrial metabolism in intact cells were published shortly after dimeric $\alpha / \beta$ tubulin was shown to block VDAC conductance in lipid bilayers and in isolated mitochondria (Rostovtseva et al., 2008). We also showed that VDAC1 and 2 isolated from VDAC double-knockdown HepG2 cells in all combinations, inserted into lipid bilayers, were equally sensitive to tubulin inhibition. By contrast, VDAC3 was insensitive at tubulin concentrations even 5-fold higher than those used to inhibit VDAC1 and 2 (Maldonado et al., 2013). In parallel with those experiments, we showed that the blockage of the inhibitory effect of tubulin on VDAC by erastin, increased mitochondrial metabolism (Maldonado et al., 2013). Erastin is a VDAC 1 and 2-binding molecule identified in a synthetic lethal chemical screening shown to induce non-apoptotic cell death (Dolma et al., 2003). Interestingly, cell death induced by erastin was blocked by antioxidants but not prevented by pan-caspase inhibitors, suggesting that erastin-induced cell death was ROS-dependent (Yagoda et al., 2007). The effect of erastin on mitochondrial metabolism was independent of the inhibition of the cystine/glutamine antiporter system $\mathrm{x}_{\mathrm{c}}^{-}$, another known target of erastin (unpublished). More recently, using a cellbased high throughput screen, we identified a series of erastinlike compounds that enhance mitochondrial metabolism, promote oxidative stress leading to mitochondrial dysfunction, and decrease glycolysis as measured by lactate release (anti-Warburg; Wright et al., 2001; DeHart et al., 2018a,b). Lately, we showed that mitochondrial dysfunction promoted by erastin/erastin-like molecules was mediated by a ROS-dependent translocation of activated JNK to mitochondria (Heslop et al., 2019). In summary, the VDAC-tubulin interaction represents a new pharmacological target to turn a pro-proliferative phenotype into a cytotoxic, mitochondrial-dependent pro-oxidant metabolism. Based on our studies on VDAC regulation in cancer, we have proposed that VDAC opening is a pro-oxidant anti-Warburg switch that promote cancer cell death (Maldonado, 2017; Fang and Maldonado, 2018; Heslop et al., 2019).

Overall, pharmacologically-induced VDAC opening, as achieved by reversal of the inhibitory effect of tubulin on VDAC, triggers two distinct and nearly simultaneous effects: 


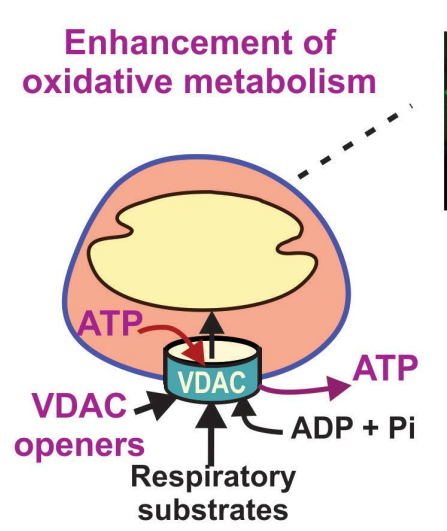

Enhancement of oxidative metabolism

substrates

\section{Increased $\Delta \Psi$ and \\ ROS formation}
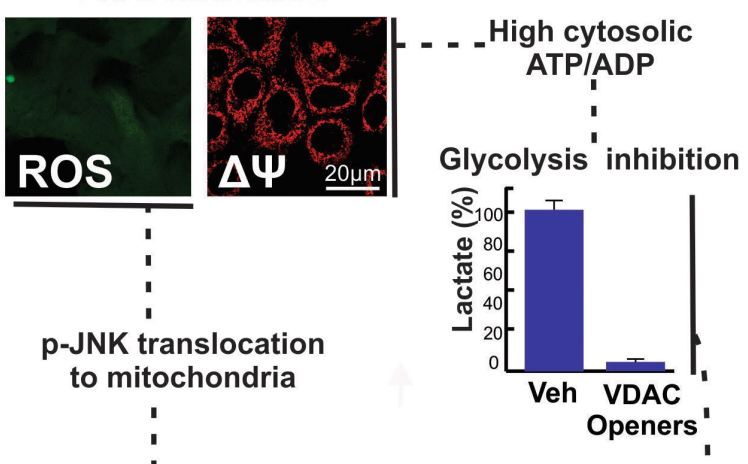

p-JNK translocation

to mitochondria

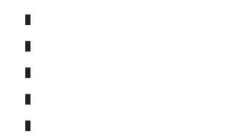

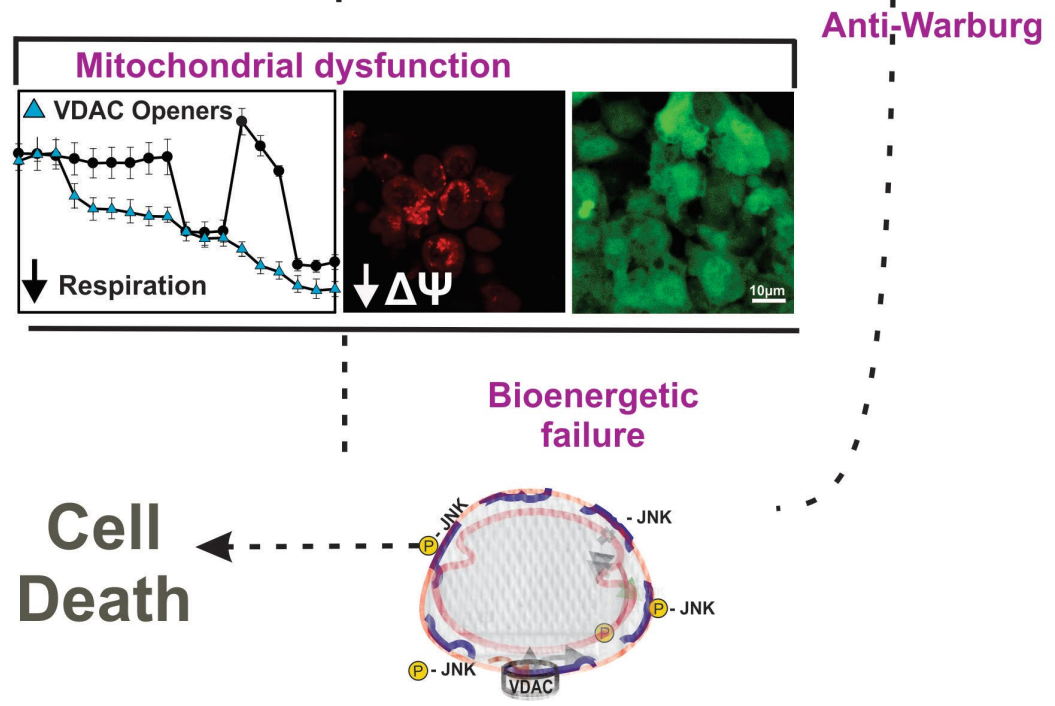

FIGURE 3 | Working model of a two-hit mechanism of cell death induced by VDAC opening. VDAC-tubulin antagonists and potentially other VDAC openers increase the flux of metabolites into and out of mitochondria. VDAC opening increases mitochondrial metabolism, promotes high cytosolic ATP/ADP ratios, increases oxygen consumption, and decreases glycolysis (anti-Warburg). VDAC opening also increases mitochondrial ROS formation causing oxidative stress and mitochondrial dysfunction. Images are from confocal fluorescence microscopy of SNU-449 hepatocarcinoma cells loaded with chloromethyl-20,70dichlorodihydrofluorescein diacetate (ROS), and tetramethyl rhodamine methyl ester $(\Delta \Psi)$.

the increase of mitochondrial metabolism and Oxphos with subsequent decrease of glycolysis (anti-Warburg), and the increase in ROS formation causing oxidative stress (Figure 3). Because of the metabolic heterogeneity of tumors, it is possible that the adverse effects of ROS accumulation and glycolysis inhibition on cell survival and proliferation be different among cells. A VDAC-dependent increase in ROS production would be likely more detrimental for highly glycolytic cells, constitutively not exposed to high levels of mitochondrial ROS. Conversely, the reversal of the Warburg effect would damage more those highly glycolytic cells that survive oxidative stress and continue proliferating, or low glycolytic cells with a presumably constitutively higher basal level of ROS. The combination of reversal of Warburg metabolism and oxidative stress by erastinlike compounds caused cell death to human hepatocarcinoma cell lines in culture and slowed tumor growth in a xenograft model of Huh7 hepatocarcinoma cells (DeHart et al., 2018a,b).
An intriguing possibility is that VDAC be implicated also in controlling metabolic fluxes in cancer stem cells (CSC), also called stem-like cancer cells, tumor-initiating cells, or cancer-initiating cells. CSC comprising $1-2 \%$ of total cells in most types of cancer, have been found in breast, lung, colon, brain, head and neck, prostate and liver tumors, among others (Batlle and Clevers, 2017). Because of the capabilities for selfrenewal, tumorigenesis, invasion, and migration, CSC are associated with high risk of metastasis and relapses after chemotherapy. CSC display either a more oxidative or a more glycolytic profile, are plastic, undergo phenotypic transitions, and depending on the tumor of origin, differentiate into non-stem tumor cells to sustain tumor growth (Fonseca et al., 2017; Gupta et al., 2019). An interesting study showed that the interaction between VDAC2 and a subunit of the phosphofructokinase 1 tetramer regulates glucose metabolism and modulates the phenotypic reprogramming of glioma stem 
cells (Zhou et al., 2018). This first work on the potential role of VDAC as a glycolytic regulator of the phenotype transition between CSC and non-stem cancer cells is opening new avenues to study VDAC as a potential therapeutic target. Although, a perspective about the role of mitochondria-tubulin interactions in the regulation of mitochondrial structure and function in CSCs has recently been reviewed, there is no actual evidence that the VDAC-tubulin interaction or VDAC opening modulates CSC plasticity and proliferation (Kim and Cheong, 2020).

\section{CONCLUDING REMARKS}

Research on different aspects of cancer bioenergetics and cancer metabolism clearly showed that tumors are metabolically heterogeneous, and that many tumors, if not all, are metabolically flexible. Moreover, the pro-proliferative Warburg phenotype can be reversed by increasing oxidative metabolism. In that regard, VDAC opening is an attractive mechanism to target pharmacologically, because it serves the dual purpose of increasing mitochondrial metabolism and ROS formation, while at the same time decreasing glycolysis. The dual action of VDAC openers as anti-Warburg and promoters of oxidative stress, represent a two-hit mechanism to induce cell death. An advantage of VDAC as a target relies on the fact that regulation of only one protein has an amplifying effect, both on mitochondrial function and subsequently on biosynthetic reactions occurring

\section{REFERENCES}

Adachi, M., Higuchi, H., Miura, S., Azuma, T., Inokuchi, S., Saito, H., et al. (2004). Bax interacts with the voltage-dependent anion channel and mediates ethanol-induced apoptosis in rat hepatocytes. Am. J. Physiol. Gastrointest. Liver Physiol. 287, G695-G705. doi: 10.1152/ajpgi.00415.2003

Al Jamal, J. A. (2005). Involvement of porin N,N-dicyclohexylcarbodiimidereactive domain in hexokinase binding to the outer mitochondrial membrane. Protein J. 24, 1-8. doi: 10.1007/s10930-004-0600-2

Alam, M. M., Lal, S., FitzGerald, K. E., and Zhang, L. (2016). A holistic view of cancer bioenergetics: mitochondrial function and respiration play fundamental roles in the development and progression of diverse tumors. Clin. Transl. Med. 5:3. doi: 10.1186/s40169-016-0082-9

Allouche, M., Pertuiset, C., Robert, J. L., Martel, C., Veneziano, R., Henry, C., et al. (2012). ANT-VDAC1 interaction is direct and depends on ANT isoform conformation in vitro. Biochem. Biophys. Res. Commun. 429, 12-17. doi: 10.1016/j.bbrc.2012.10.108

Baines, C. P., Kaiser, R. A., Sheiko, T., Craigen, W. J., and Molkentin, J. D. (2007). Voltage-dependent anion channels are dispensable for mitochondrial-dependent cell death. Nat. Cell Biol. 9, 550-555. doi: 10.1038/ ncb1575

Barcena-Varela, M., and Lujambio, A. (2021). The endless sources of hepatocellular carcinoma heterogeneity. Cancer 13:2621. doi: 10.3390/cancers13112621

Batlle, E., and Clevers, H. (2017). Cancer stem cells revisited. Nat. Med. 23, 1124-1134. doi: $10.1038 / \mathrm{nm} .4409$

Bera, A. K., Ghosh, S., and Das, S. (1995). Mitochondrial VDAC can be phosphorylated by cyclic AMP-dependent protein kinase. Biochem. Biophys. Res. Commun. 209, 213-217. doi: 10.1006/bbrc.1995.1491

Bhat, T. A., Kumar, S., Chaudhary, A. K., Yadav, N., and Chandra, D. (2015). Restoration of mitochondria function as a target for cancer therapy. Drug Discov. Today 20, 635-643. doi: 10.1016/j.drudis.2015.03.001

Bohm, R., Amodeo, G. F., Murlidaran, S., Chavali, S., Wagner, G., Winterhalter, M., et al. (2020). The structural basis for low conductance in the membrane in the cytosol, which depend on mitochondrial intermediaries. As it was developed here, the understanding of endogenous mechanisms regulating VDAC conformational states is relevant for cancer biology and for potential pharmacological applications. The search for drugs promoting VDAC opening directly, or indirectly by blocking endogenous regulators like is the case for VDAC-tubulin, open a new era in the development of novel metabolism-oriented cancer chemotherapy.

\section{AUTHOR CONTRIBUTIONS}

$\mathrm{KH}$ contributed with the search for updated literature, compilation of sources, writing and figures preparation. VM contributed with writing and preparation of Figure 2. EM organized the structure of the review, intervened in all the steps of the review preparation, contributed to the writing, and made final adjustments to the text and figures. All authors contributed to the article and approved the submitted version.

\section{FUNDING}

This work was supported by NIH grant NCI R01CA184456 and SCTR Pilot Project UL1 TR001450-SCTR to EM; and the Abney Foundation Fellowship from MUSC Hollings Cancer Center to $\mathrm{KH}$.

protein VDAC upon beta-NADH binding and voltage gating. Structure 28, 206.e4-214.e4. doi: 10.1016/j.str.2019.11.015

Bott, A. J., Maimouni, S., and Zong, W. X. (2019). The pleiotropic effects of glutamine metabolism in cancer. Cancer 11:770. doi: 10.3390/cancers 11060770

Bowen, K. A., Tam, K., and Colombini, M. (1985). Evidence for titratable gating charges controlling the voltage dependence of the outer mitochondrial membrane channel, VDAC. J. Membr. Biol. 86, 51-59. doi: 10.1007/ BF01871610

Brahimi-Horn, M. C., Ben-Hail, D., Ilie, M., Gounon, P., Rouleau, M., Hofman, V., et al. (2012). Expression of a truncated active form of VDAC1 in lung cancer associates with hypoxic cell survival and correlates with progression to chemotherapy resistance. Cancer Res. 72, 2140-2150. doi: 10.1158/0008-5472. CAN-11-3940

Brahimi-Horn, M. C., Giuliano, S., Saland, E., Lacas-Gervais, S., Sheiko, T., Pelletier, J., et al. (2015). Knockout of Vdacl activates hypoxia-inducible factor through reactive oxygen species generation and induces tumor growth by promoting metabolic reprogramming and inflammation. Cancer Metab. 3:8. doi: 10.1186/s40170-015-0133-5

Brahimi-Horn, M. C., and Mazure, N. M. (2014). Hypoxic VDAC1: a potential mitochondrial marker for cancer therapy. Adv. Exp. Med. Biol. 772, 101-110. doi: 10.1007/978-1-4614-5915-6 5

Brand, M. D. (2005). The efficiency and plasticity of mitochondrial energy transduction. Biochem. Soc. Trans. 33, 897-904. doi: 10.1042/ BST0330897

Budelier, M. M., Cheng, W. W. L., Bergdoll, L., Chen, Z. W., Janetka, J. W., Abramson, J., et al. (2017). Photoaffinity labeling with cholesterol analogues precisely maps a cholesterol-binding site in voltage-dependent anion channel-1. J. Biol. Chem. 292, 9294-9304. doi: 10.1074/jbc.M116.773069

Camara, A. K. S., Zhou, Y., Wen, P. C., Tajkhorshid, E., and Kwok, W. M. (2017). Mitochondrial VDAC1: a key gatekeeper as potential therapeutic target. Front. Physiol. 8:460. doi: 10.3389/fphys.2017.00460

Cerezo, M., and Rocchi, S. (2020). Cancer cell metabolic reprogramming: a keystone for the response to immunotherapy. Cell Death Dis. 11:964. doi: $10.1038 /$ s41419-020-03175-5 
Chance, B., Sies, H., and Boveris, A. (1979). Hydroperoxide metabolism in mammalian organs. Physiol. Rev. 59, 527-605. doi: 10.1152/ physrev.1979.59.3.527

Chin, H. S., Li, M. X., Tan, I. K. L., Ninnis, R. L., Reljic, B., Scicluna, K., et al. (2018). VDAC2 enables BAX to mediate apoptosis and limit tumor development. Nat. Commun. 9:4976. doi: 10.1038/s41467-018-07309-4

Choudhary, O. P., Ujwal, R., Kowallis, W., Coalson, R., Abramson, J., and Grabe, M. (2010). The electrostatics of VDAC: implications for selectivity and gating. J. Mol. Biol. 396, 580-592. doi: 10.1016/j.jmb.2009.12.006

Clavell, L. A., Gelber, R. D., Cohen, H. J., Hitchcock-Bryan, S., Cassady, J. R., Tarbell, N. J., et al. (1986). Four-agent induction and intensive asparaginase therapy for treatment of childhood acute lymphoblastic leukemia. N. Engl. J. Med. 315, 657-663. doi: 10.1056/NEJM198609113151101

Colombini, M. (1979). A candidate for the permeability pathway of the outer mitochondrial membrane. Nature 279, 643-645. doi: 10.1038/279643a0

Colombini, M. (1980). Structure and mode of action of a voltage dependent anion-selective channel (VDAC) located in the outer mitochondrial membrane. Ann. N. Y. Acad. Sci. 341, 552-563. doi: 10.1111/j.1749-6632.1980.tb47198.x

Colombini, M. (1989). Voltage gating in the mitochondrial channel, VDAC. J. Membr. Biol. 111, 103-111. doi: 10.1007/BF01871775

Colombini, M. (2004). VDAC: the channel at the interface between mitochondria and the cytosol. Mol. Cell Biochem. 256-257, 107-115. doi: 10.1023/b:mcbi.0000009862.17396.8d.

Colombini, M. (2009). The published 3D structure of the VDAC channel: native or not? Trends Biochem. Sci. 34, 382-389. doi: 10.1016/j.tibs.2009.05.001

Colombini, M. (2012). VDAC structure, selectivity, and dynamics. Biochim. Biophys. Acta 1818, 1457-1465. doi: 10.1016/j.bbamem.2011.12.026

Colombini, M. (2016). The VDAC channel: molecular basis for selectivity. Biochim. Biophys. Acta 1863, 2498-2502. doi: 10.1016/j.bbamcr.2016.01.019

Comerford, S. A., Huang, Z., Du, X., Wang, Y., Cai, L., Witkiewicz, A. K., et al. (2014). Acetate dependence of tumors. Cell 159, 1591-1602. doi: 10.1016/j.cell.2014.11.020

Commisso, C., Davidson, S. M., Soydaner-Azeloglu, R. G., Parker, S. J., Kamphorst, J. J., Hackett, S., et al. (2013). Macropinocytosis of protein is an amino acid supply route in Ras-transformed cells. Nature 497, 633-637. doi: $10.1038 /$ nature 12138

Cunha-de Padua, M. M., Fabbri, L., Dufies, M., Lacas-Gervais, S., Contenti, J., Voyton, C., et al. (2020). Evidences of a direct relationship between cellular fuel supply and ciliogenesis regulated by hypoxic VDAC1-DeltaC. Cancer 12:3484. doi: 10.3390/cancers12113484

Curcio, C., Brugiapaglia, S., Bulfamante, S., Follia, L., Cappello, P., and Novelli, F. (2021). The glycolytic pathway as a target for novel onco-immunology therapies in pancreatic cancer. Molecules 26:1642. doi: 10.3390/ molecules26061642

Das, S., Wong, R., Rajapakse, N., Murphy, E., and Steenbergen, C. (2008). Glycogen synthase kinase 3 inhibition slows mitochondrial adenine nucleotide transport and regulates voltage-dependent anion channel phosphorylation. Circ. Res. 103, 983-991. doi: 10.1161/CIRCRESAHA.108.178970

De Pinto, V., Guarino, F., Guarnera, A., Messina, A., Reina, S., Tomasello, F. M., et al. (2010). Characterization of human VDAC isoforms: a peculiar function for VDAC3? Biochim. Biophys. Acta 1797, 1268-1275. doi: 10.1016/j. bbabio.2010.01.031

DeBerardinis, R. J., and Chandel, N. S. (2020). We need to talk about the Warburg effect. Nat. Metab. 2, 127-129. doi: 10.1038/s42255-020-0172-2

DeHart, D. N., Fang, D., Heslop, K., Li, L., Lemasters, J. J., and Maldonado, E. N. (2018a). Opening of voltage dependent anion channels promotes reactive oxygen species generation, mitochondrial dysfunction and cell death in cancer cells. Biochem. Pharmacol. 148, 155-162. doi: 10.1016/j. bcp.2017.12.022

DeHart, D. N., Lemasters, J. J., and Maldonado, E. N. (2018b). Erastin-like anti-Warburg agents prevent mitochondrial depolarization induced by free tubulin and decrease lactate formation in cancer cells. SLAS Discov. 23, 23-33. doi: 10.1177/2472555217731556

Doherty, J. R., and Cleveland, J. L. (2013). Targeting lactate metabolism for cancer therapeutics. J. Clin. Invest. 123, 3685-3692. doi: 10.1172/JCI69741

Dolma, S., Lessnick, S. L., Hahn, W. C., and Stockwell, B. R. (2003). Identification of genotype-selective antitumor agents using synthetic lethal chemical screening in engineered human tumor cells. Cancer Cell 3, 285-296. doi: 10.1016/ S1535-6108(03)00050-3
Duraj, T., Garcia-Romero, N., Carrion-Navarro, J., Madurga, R., Mendivil, A. O., Prat-Acin, R., et al. (2021). Beyond the Warburg effect: oxidative and glycolytic phenotypes coexist within the metabolic heterogeneity of glioblastoma. Cells 10:202. doi: $10.3390 /$ cells 10020202

Fan, M., Zhang, J., Tsai, C. W., Orlando, B. J., Rodriguez, M., Xu, Y., et al. (2020). Structure and mechanism of the mitochondrial $\mathrm{ca}(2+)$ uniporter holocomplex. Nature 582, 129-133. doi: 10.1038/s41586-020-2309-6

Fang, D., and Maldonado, E. N. (2018). VDAC regulation: a mitochondrial target to stop cell proliferation. Adv. Cancer Res. 138, 41-69. doi: 10.1016/ bs.acr.2018.02.002

Faubert, B., Solmonson, A., and DeBerardinis, R. J. (2020). Metabolic reprogramming and cancer progression. Science 368:eaaw5473. doi: 10.1126/ science.aaw5473

Fonseca, N. A., Cruz, A. F., Moura, V., Simoes, S., and Moreira, J. N. (2017). The cancer stem cell phenotype as a determinant factor of the heterotypic nature of breast tumors. Crit. Rev. Oncol. Hematol. 113, 111-121. doi: 10.1016/j.critrevonc.2017.03.016

Gambardella, J., Lombardi, A., and Santulli, G. (2020). Metabolic flexibility of mitochondria plays a key role in balancing glucose and fatty acid metabolism in the diabetic heart. Diabetes 69, 2054-2057. doi: 10.2337/dbi20-0024

Gentric, G., Mieulet, V., and Mechta-Grigoriou, F. (2017). Heterogeneity in cancer metabolism: new concepts in an old field. Antioxid Redox Signal. 26, 462-485. doi: 10.1089/ars.2016.6750

Gincel, D., Zaid, H., and Shoshan-Barmatz, V. (2001). Calcium binding and translocation by the voltage-dependent anion channel: a possible regulatory mechanism in mitochondrial function. Biochem. J. 358, 147-155. doi: 10.1042/ bj3580147

Griguer, C. E., Oliva, C. R., and Gillespie, G. Y. (2005). Glucose metabolism heterogeneity in human and mouse malignant glioma cell lines. J. NeuroOncol. 74, 123-133. doi: 10.1007/s11060-004-6404-6

Grills, C., Jithesh, P. V., Blayney, J., Zhang, S. D., and Fennell, D. A. (2011). Gene expression meta-analysis identifies VDAC1 as a predictor of poor outcome in early stage non-small cell lung cancer. PLoS One 6:e14635. doi: 10.1371/journal.pone.0014635

Gupta, P. B., Pastushenko, I., Skibinski, A., Blanpain, C., and Kuperwasser, C. (2019). Phenotypic plasticity: driver of cancer initiation, progression, and therapy resistance. Cell Stem Cell 24, 65-78. doi: 10.1016/j. stem.2018.11.011

Heiden, M. G. V., Li, X. X., Gottleib, E., Hill, R. B., Thompson, C. B., and Colombini, M. (2001). Bcl-xL promotes the open configuration of the voltage-dependent anion channel and metabolite passage through the outer mitochondrial membrane. J. Biol. Chem. 276, 19414-19419. doi: 10.1074/ jbc.M101590200

Heslop, K. A., Rovini, A., Hunt, E. G., Fang, D., Morris, M. E., Christie, C. F., et al. (2019). JNK activation and translocation to mitochondria mediates mitochondrial dysfunction and cell death induced by VDAC opening and sorafenib in hepatocarcinoma cells. Biochem. Pharmacol. 171:113728. doi: 10.1016/j.bcp.2019.113728

Hiller, S., Abramson, J., Mannella, C., Wagner, G., and Zeth, K. (2010). The 3D structures of VDAC represent a native conformation. Trends Biochem. Sci. 35, 514-521. doi: 10.1016/j.tibs.2010.03.005

Hoek, J. B., Nicholls, D. G., and Williamson, J. R. (1980). Determination of the mitochondrial protonmotive force in isolated hepatocytes. J. Biol. Chem. 255, 1458-1464. doi: 10.1016/S0021-9258(19)86052-0

Jenkins, C. M., Yang, J., Sims, H. F., and Gross, R. W. (2011). Reversible high affinity inhibition of phosphofructokinase-1 by acyl-CoA: a mechanism integrating glycolytic flux with lipid metabolism. J. Biol. Chem. 286, 1193711950. doi: 10.1074/jbc.M110.203661

Jose, C., Bellance, N., and Rossignol, R. (2011). Choosing between glycolysis and oxidative phosphorylation: a tumor's dilemma? Biochim. Biophys. Acta 1807, 552-561. doi: 10.1016/j.bbabio.2010.10.012

Jozwiak, P., Ciesielski, P., Forma, E., Kozal, K., Wojcik-Krowiranda, K., Cwonda, L., et al. (2020). Expression of voltage-dependent anion channels in endometrial cancer and its potential prognostic significance. Tumour Biol. 42:10104 28320951057. doi: 10.1177/1010428320951057

Kanwar, P., Samtani, H., Sanyal, S. K., Srivastava, A. K., Suprasanna, P., and Pandey, G. K. (2020). VDAC and its interacting partners in plant and animal systems: an overview. Crit. Rev. Biotechnol. 40, 715-732. doi: $10.1080 / 07388551.2020 .1756214$ 
Keenan, M. M., and Chi, J. T. (2015). Alternative fuels for cancer cells. Cancer J. 21, 49-55. doi: 10.1097/PPO.0000000000000104

Kelley, D. E., Goodpaster, B., Wing, R. R., and Simoneau, J. A. (1999). Skeletal muscle fatty acid metabolism in association with insulin resistance, obesity, and weight loss. Am. J. Physiol. 277, E1130-E1141. doi: 10.1152/ ajpendo.1999.277.6.E1130

Kelley, D. E., and Simoneau, J. A. (1994). Impaired free fatty acid utilization by skeletal muscle in non-insulin-dependent diabetes mellitus. J. Clin. Invest. 94, 2349-2356. doi: 10.1172/JCI117600

Kennedy, K. M., Scarbrough, P. M., Ribeiro, A., Richardson, R., Yuan, H., Sonveaux, P., et al. (2013). Catabolism of exogenous lactate reveals it as a legitimate metabolic substrate in breast cancer. PLoS One 8:e75154. doi: 10.1371/journal.pone.0075154

Kiesel, V. A., Sheeley, M. P., Coleman, M. F., Cotul, E. K., Donkin, S. S., Hursting, S. D., et al. (2021). Pyruvate carboxylase and cancer progression. Cancer Metab. 9:20. doi: 10.1186/s40170-021-00256-7

Kilburn, D. G., Lilly, M. D., and Webb, F. C. (1969). The energetics of mammalian cell growth. J. Cell Sci. 4, 645-654.

Kim, J., and Cheong, J. H. (2020). Role of mitochondria-cytoskeleton interactions in the regulation of mitochondrial structure and function in cancer stem cells. Cells 9:1691. doi: 10.3390/cells9071691

Kim, J., and DeBerardinis, R. J. (2019). Mechanisms and implications of metabolic heterogeneity in cancer. Cell Metab. 30, 434-446. doi: 10.1016/j. cmet.2019.08.013

Klingenberg, M. (2008). The ADP and ATP transport in mitochondria and its carrier. Biochim. Biophys. Acta 1778, 1978-2021. doi: 10.1016/j.bbamem. 2008.04.011

Kreis, W., Baker, A., Ryan, V., and Bertasso, A. (1980). Effect of nutritional and enzymatic methionine deprivation upon human normal and malignant cells in tissue culture. Cancer Res. 40, 634-641.

Kuipers, J., Jahn, K., and Beerenwinkel, N. (2017). Advances in understanding tumour evolution through single-cell sequencing. Biochim. Biophys. Acta Rev. Cancer 1867, 127-138. doi: 10.1016/j.bbcan.2017.02.001

Lemeshko, V. V. (2021). Electrical control of the cell energy metabolism at the level of mitochondrial outer membrane. Biochim. Biophys. Acta Biomembr. 1863:183493. doi: 10.1016/j.bbamem.2020.183493

Li, T., and Le, A. (2018). Glutamine metabolism in cancer. Adv. Exp. Med. Biol. 1063, 13-32. doi: 10.1007/978-3-319-77736-8_2

Lim, H. Y., Ho, Q. S., Low, J., Choolani, M., and Wong, K. P. (2011). Respiratory competent mitochondria in human ovarian and peritoneal cancer. Mitochondrion 11, 437-443. doi: 10.1016/j.mito.2010.12.015

Magri, A., Reina, S., and De Pinto, V. (2018). VDAC1 as pharmacological target in cancer and neurodegeneration: focus on its role in apoptosis. Front. Chem. 6:108. doi: 10.3389/fchem.2018.00108

Mailloux, R. J. (2020). An update on mitochondrial reactive oxygen species production. Antioxidants 9:472. doi: 10.3390/antiox9060472

Maldonado, E. N. (2017). VDAC-tubulin, an anti-Warburg pro-oxidant switch. Front. Oncol. 7:4. doi: 10.3389/fonc.2017.00004

Maldonado, E. N., Patnaik, J., Mullins, M. R., and Lemasters, J. J. (2010). Free tubulin modulates mitochondrial membrane potential in cancer cells. Cancer Res. 70, 10192-10201. doi: 10.1158/0008-5472.CAN-10-2429

Maldonado, E. N., Sheldon, K. L., DeHart, D. N., Patnaik, J., Manevich, Y., Townsend, D. M., et al. (2013). Voltage-dependent anion channels modulate mitochondrial metabolism in cancer cells: regulation by free tubulin and erastin. J. Biol. Chem. 288, 11920-11929. doi: 10.1074/jbc.M112.433847

Mannella, C. A. (2021). VDAC-A primal perspective. Int. J. Mol. Sci. 22:1685. doi: $10.3390 /$ ijms 22041685

Martins, F., Goncalves, L. G., Pojo, M., and Serpa, J. (2020). Take advantage of glutamine anaplerosis, the kernel of the metabolic rewiring in malignant gliomas. Biomolecules 10:1370. doi: 10.3390/biom10101370

Mathupala, S. P., Ko, Y. H., and Pedersen, P. L. (2010). The pivotal roles of mitochondria in cancer: Warburg and beyond and encouraging prospects for effective therapies. Biochim. Biophys. Acta 1797, 1225-1230. doi: 10.1016/j. bbabio.2010.03.025

Mazure, N. M. (2016). News about VDAC1 in hypoxia. Front. Oncol. 6:193. doi: $10.3389 /$ fonc. 2016.00193

McKeown, S. R. (2014). Defining normoxia, physoxia and hypoxia in tumoursimplications for treatment response. Br. J. Radiol. 87:20130676. doi: 10.1259/ bjr.20130676
Meiser, J., and Vazquez, A. (2016). Give it or take it: the flux of one-carbon in cancer cells. FEBS J. 283, 3695-3704. doi: 10.1111/febs.13731

Mitchell, P. (1966). Chemiosmotic coupling in oxidative and photosynthetic phosphorylation. Biol. Rev. Camb. Philos. Soc. 41, 445-502. doi: 10.1111/j. 1469-185X.1966.tb01501.x

Mitchell, P., and Moyle, J. (1967). Chemiosmotic hypothesis of oxidative phosphorylation. Nature 213, 137-139. doi: 10.1038/213137a0

Moreno-Sanchez, R., Marin-Hernandez, A., Saavedra, E., Pardo, J. P., Ralph, S. J., and Rodriguez-Enriquez, S. (2014). Who controls the ATP supply in cancer cells? Biochemistry lessons to understand cancer energy metabolism. Int. J. Biochem. Cell Biol. 50, 10-23. doi: 10.1016/j.biocel.2014.01.025

Motori, E., Atanassov, I., Kochan, S. M. V., Folz-Donahue, K., Sakthivelu, V., Giavalisco, P., et al. (2020). Neuronal metabolic rewiring promotes resilience to neurodegeneration caused by mitochondrial dysfunction. Sci. Adv. 6:eaba8271. doi: $10.1126 /$ sciadv.aba8271

Mullen, A. R., Wheaton, W. W., Jin, E. S., Chen, P. H., Sullivan, L. B., Cheng, T., et al. (2012). Reductive carboxylation supports growth in tumour cells with defective mitochondria. Nature 481, 385-388. doi: 10.1038/nature10642

Nakashima, R. A., Paggi, M. G., and Pedersen, P. L. (1984). Contributions of glycolysis and oxidative phosphorylation to adenosine $5^{\prime}$-triphosphate production in AS-30D hepatoma cells. Cancer Res. 44, 5702-5706.

Nicholls, D. G., and Ferguson, S. J. (2013). Bioenergetics. London: Elsevier.

Owen, O. E., Kalhan, S. C., and Hanson, R. W. (2002). The key role of anaplerosis and cataplerosis for citric acid cycle function. J. Biol. Chem. 277, 30409-30412. doi: $10.1074 /$ jbc.R200006200

Palmieri, F., and Pierri, C. L. (2010). Mitochondrial metabolite transport. Essays Biochem. 47, 37-52. doi: 10.1042/bse0470037

Pastorino, J. G., Shulga, N., and Hoek, J. B. (2002). Mitochondrial binding of hexokinase II inhibits bax-induced cytochrome $\mathrm{c}$ release and apoptosis. $J$. Biol. Chem. 277, 7610-7618. doi: 10.1074/jbc.M109950200

Porcelli, A. M., Ghelli, A., Zanna, C., Pinton, P., Rizzuto, R., and Rugolo, M. (2005). pH difference across the outer mitochondrial membrane measured with a green fluorescent protein mutant. Biochem. Biophys. Res. Commun. 326, 799-804. doi: 10.1016/j.bbrc.2004.11.105

Rich, P. R., and Marechal, A. (2010). The mitochondrial respiratory chain. Essays Biochem. 47, 1-23. doi: 10.1042/bse0470001

Robinson, G. L., Dinsdale, D., Macfarlane, M., and Cain, K. (2012). Switching from aerobic glycolysis to oxidative phosphorylation modulates the sensitivity of mantle cell lymphoma cells to TRAIL. Oncogene 31, 4996-5006. doi: 10.1038/onc.2012.13

Rodriguez-Enriquez, S., Carreno-Fuentes, L., Gallardo-Perez, J. C., Saavedra, E., Quezada, H., Vega, A., et al. (2010). Oxidative phosphorylation is impaired by prolonged hypoxia in breast and possibly in cervix carcinoma. Int. J. Biochem. Cell Biol. 42, 1744-1751. doi: 10.1016/j.biocel.2010.07.010

Rostovtseva, T. K., Queralt-Martin, M., Rosencrans, W. M., and Bezrukov, S. M. (2020). Targeting the multiple physiologic roles of VDAC with steroids and hydrophobic drugs. Front. Physiol. 11:446. doi: 10.3389/ fphys.2020.00446

Rostovtseva, T. K., Sheldon, K. L., Hassanzadeh, E., Monge, C., Saks, V., Bezrukov, S. M., et al. (2008). Tubulin binding blocks mitochondrial voltagedependent anion channel and regulates respiration. Proc. Natl. Acad. Sci. U. S. A. 105, 18746-18751. doi: 10.1073/pnas.0806303105

Rovini, A. (2019). Tubulin-VDAC interaction: molecular basis for mitochondrial dysfunction in chemotherapy-induced peripheral neuropathy. Front. Physiol. 10:671. doi: 10.3389/fphys.2019.00671

Sampson, M. J., Lovell, R. S., and Craigen, W. J. (1997). The murine voltagedependent anion channel gene family. Conserved structure and function. J. Biol. Chem. 272, 18966-18973. doi: 10.1074/jbc.272.30.18966

Sander, P., Gudermann, T., and Schredelseker, J. (2021). A calcium guard in the outer membrane: is VDAC a regulated gatekeeper of mitochondrial calcium uptake? Int. J. Mol. Sci. 22:946. doi: 10.3390/ijms22020946

Schein, S. J., Colombini, M., and Finkelstein, A. (1976). Reconstitution in planar lipid bilayers of a voltage-dependent anion-selective channel obtained from paramecium mitochondria. J. Membr. Biol. 30, 99-120. doi: 10.1007/ BF01869662

Schwenke, W. D., Soboll, S., Seitz, H. J., and Sies, H. (1981). Mitochondrial and cytosolic ATP/ADP ratios in rat liver in vivo. Biochem. J. 200, 405-408. doi: $10.1042 / b j 2000405$ 
Scott, L., Lamb, J., Smith, S., and Wheatley, D. N. (2000). Single amino acid (arginine) deprivation: rapid and selective death of cultured transformed and malignant cells. Br. J. Cancer 83, 800-810. doi: 10.1054/bjoc.2000.1353

Seyfried, T. N., Arismendi-Morillo, G., Mukherjee, P., and Chinopoulos, C. (2020). On the Origin of ATP Synthesis in Cancer. iScience 23, 101761. doi: $10.1016 /$ j.isci.2020.101761

Sheldon, K. L., Maldonado, E. N., Lemasters, J. J., Rostovtseva, T. K., and Bezrukov, S. M. (2011). Phosphorylation of voltage-dependent anion channel by serine/threonine kinases governs its interaction with tubulin. PLoS One 6:e25539. doi: 10.1371/journal.pone.0025539

Shevade, A., Strogolova, V., Orlova, M., Yeo, C. T., and Kuchin, S. (2018). Mitochondrial voltage-dependent anion channel protein porl positively regulates the nuclear localization of saccharomyces cerevisiae AMP-activated protein kinase. mSphere 3, e00482-e00517. doi: 10.1128/mSphere.00482-17

Shoshan-Barmatz, V., Maldonado, E. N., and Krelin, Y. (2017). VDAC1 at the crossroads of cell metabolism, apoptosis and cell stress. Cell Stress 1, 11-36. doi: $10.15698 /$ cst2017.10.104

Shuvo, S. R., Ferens, F. G., and Court, D. A. (2016). The N-terminus of VDAC: structure, mutational analysis, and a potential role in regulating barrel shape. Biochim. Biophys. Acta 1858, 1350-1361. doi: 10.1016/j.bbamem.2016.03.017

Singleterry, J., Sreedhar, A., and Zhao, Y. (2014). Components of cancer metabolism and therapeutic interventions. Mitochondrion 17C, 50-55. doi: 10.1016/j.mito.2014.05.010

Smolkova, K., Bellance, N., Scandurra, F., Genot, E., Gnaiger, E., Plecita-Hlavata, L., et al. (2010). Mitochondrial bioenergetic adaptations of breast cancer cells to aglycemia and hypoxia. J. Bioenerg. Biomembr. 42, 55-67. doi: 10.1007/ s10863-009-9267-x

Spinelli, J. B., and Haigis, M. C. (2018). The multifaceted contributions of mitochondria to cellular metabolism. Nat. Cell Biol. 20, 745-754. doi: 10.1038/ s41556-018-0124-1

Still, E. R., and Yuneva, M. O. (2017). Hopefully devoted to Q: targeting glutamine addiction in cancer. Br. J. Cancer 116, 1375-1381. doi: 10.1038/ bjc.2017.113

Tan, W., and Colombini, M. (2007). VDAC closure increases calcium ion flux. Biochim. Biophys. Acta 1768, 2510-2515. doi: 10.1016/j.bbamem.2007.06.002

Timohhina, N., Guzun, R., Tepp, K., Monge, C., Varikmaa, M., Vija, H., et al. (2009). Direct measurement of energy fluxes from mitochondria into cytoplasm in permeabilized cardiac cells in situ: some evidence for mitochondrial interactosome. J. Bioenerg. Biomembr. 41, 259-275. doi: 10.1007/ s10863-009-9224-8

Trepanowski, J. F., Canale, R. E., Marshall, K. E., Kabir, M. M., and Bloomer, R. J. (2011). Impact of caloric and dietary restriction regimens on markers of health and longevity in humans and animals: a summary of available findings. Nutr. J. 10:107. doi: 10.1186/1475-2891-10-107

Tsujimoto, Y., and Shimizu, S. (2000). VDAC regulation by the Bcl-2 family of proteins. Cell Death Differ. 7, 1174-1181. doi: 10.1038/sj.cdd.4400780

Ujwal, R., Cascio, D., Colletier, J. P., Faham, S., Zhang, J., Toro, L., et al. (2008). The crystal structure of mouse VDAC1 at $2.3 \mathrm{~A}$ resolution reveals mechanistic insights into metabolite gating. Proc. Natl. Acad. Sci. U. S. A. 105, 17742-17747. doi: 10.1073/pnas.0809634105

Vaupel, P., Kelleher, D. K., and Hockel, M. (2001). Oxygen status of malignant tumors: pathogenesis of hypoxia and significance for tumor therapy. Semin. Oncol. 28, 29-35. doi: 10.1016/S0093-7754(01)90210-6

Veech, R. L., King, M. T., Pawlosky, R., Bradshaw, P. C., and Curtis, W. (2019). Relationship between inorganic ion distribution, resting membrane potential, and the $\Delta G^{\prime}$ of ATP hydrolysis: a new paradigm. FASEB J. 33, 13126-13130. doi: 10.1096/fi.201901942R

Villinger, S., Giller, K., Bayrhuber, M., Lange, A., Griesinger, C., Becker, S., et al. (2014). Nucleotide interactions of the human voltage-dependent anion channel. J. Biol. Chem. 289, 13397-13406. doi: 10.1074/jbc.M113.524173
Walker, J. E. (2013). The ATP synthase: the understood, the uncertain and the unknown. Biochem. Soc. Trans. 41, 1-16. doi: 10.1042/BST20110773

Warburg, O. (1930). Ueber den stoffwechsel der tumoren. London: Constable. Warburg, O. (1956). On the origin of cancer cells. Science 123, 309-314. doi: 10.1126/science.123.3191.309

Warburg, O., Wind, F., and Negelein, E. (1927). The metabolism of tumors in the body. J. Gen. Physiol. 8, 519-530. doi: 10.1085/jgp.8.6.519

Wei, Y., Huang, C. X., Xiao, X., Chen, D. P., Shan, H., He, H., et al. (2021). $B$ cell heterogeneity, plasticity, and functional diversity in cancer microenvironments. Oncogene 40, 4737-4745. doi: 10.1038/s41388-021-01918-y

Weinberg, S. E., and Chandel, N. S. (2015). Targeting mitochondria metabolism for cancer therapy. Nat. Chem. Biol. 11, 9-15. doi: 10.1038/nchembio.1712 Weinhouse, S. (1956). On respiratory impairment in cancer cells. Science 124, 267-269. doi: 10.1126/science.124.3215.267

Wersall, O. C., Lofstedt, L., Govorov, I., Mints, M., Gabrielson, M., and Shoshan, M. (2021). PGClalpha and VDAC1 expression in endometrial cancer. Mol. Clin. Oncol. 14:42. doi: 10.3892/mco.2020.2203

Wikstrom, M., Sharma, V., Kaila, V. R., Hosler, J. P., and Hummer, G. (2015). New perspectives on proton pumping in cellular respiration. Chem. Rev. 115, 2196-2221. doi: $10.1021 / \mathrm{cr} 500448 \mathrm{t}$

Wright, G., Terada, K., Yano, M., Sergeev, I., and Mori, M. (2001). Oxidative stress inhibits the mitochondrial import of preproteins and leads to their degradation. Exp. Cell Res. 263, 107-117. doi: 10.1006/excr.2000.5096

Yagoda, N., von Rechenberg, M., Zaganjor, E., Bauer, A. J., Yang, W. S., Fridman, D. J., et al. (2007). RAS-RAF-MEK-dependent oxidative cell death involving voltagedependent anion channels. Nature 447, 864-868. doi: 10.1038/nature05859

Yang, G., Zhou, D., Li, J., Wang, W., Zhong, W., Fan, W., et al. (2019). VDAC1 is regulated by $\mathrm{BRD} 4$ and contributes to JQ1 resistance in breast cancer. Oncol. Lett. 18, 2340-2347. doi: 10.3892/ol.2019.10534

Zhou, K., Yao, Y. L., He, Z. C., Chen, C., Zhang, X. N., Yang, K. D., et al. (2018). VDAC2 interacts with PFKP to regulate glucose metabolism and phenotypic reprogramming of glioma stem cells. Cell Death Dis. 9:988. doi: 10.1038/s41419-018-1015-x

Zhu, A., Lee, D., and Shim, H. (2011). Metabolic positron emission tomography imaging in cancer detection and therapy response. Semin. Oncol. 38, 55-69. doi: 10.1053/j.seminoncol.2010.11.012

Zizi, M., Byrd, C., Boxus, R., and Colombini, M. (1998). The voltage-gating process of the voltage-dependent anion channel is sensitive to ion flow. Biophys. J. 75, 704-713. doi: 10.1016/S0006-3495(98)77560-5

Zizi, M., Forte, M., Blachly-Dyson, E., and Colombini, M. (1994). NADH regulates the gating of VDAC, the mitochondrial outer membrane channel J. Biol. Chem. 269, 1614-1616. doi: 10.1016/S0021-9258(17)42070-9

Conflict of Interest: The authors declare that the research was conducted in the absence of any commercial or financial relationships that could be construed as a potential conflict of interest.

Publisher's Note: All claims expressed in this article are solely those of the authors and do not necessarily represent those of their affiliated organizations, or those of the publisher, the editors and the reviewers. Any product that may be evaluated in this article, or claim that may be made by its manufacturer, is not guaranteed or endorsed by the publisher.

Copyright (๑) 2021 Heslop, Milesi and Maldonado. This is an open-access article distributed under the terms of the Creative Commons Attribution License (CC BY). The use, distribution or reproduction in other forums is permitted, provided the original author(s) and the copyright owner(s) are credited and that the original publication in this journal is cited, in accordance with accepted academic practice. No use, distribution or reproduction is permitted which does not comply with these terms. 